\title{
Traumaspezifisches Wissen auch für Suchttherapeuten!
}

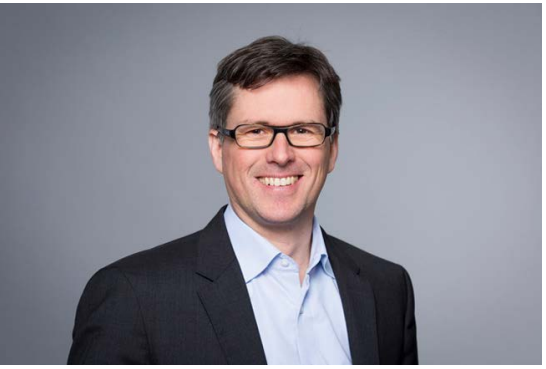

Über Trauma- und Suchtstörungen sowie über Therapiemöglichkeiten sprach Prof. Dr. Michael Klein (Köln) mit dem Experten Prof. Dr. Ingo Schäfer (Hamburg).

Wie kamen Sie in Ihrer Karriere persönlich auf das Thema Trauma und Sucht?

Prof. Schäfer: Zum Thema Trauma kam ich durch meine Doktorarbeit, die ich Ende der 90er Jahre zu Traumatisierungen bei Kindern und Jugendlichen verfasste. Als ich etwas später begann mit erwachsenen Patientinnen und Patienten in der Psychiatrie zu arbeiten haben mich zwei Dinge überrascht. Zum einen deren hohe Belastung mit frühen Traumatisierungen, zum anderen, wie wenig dies Thema in der Psychiatrie war. Zusammen mit einigen Kolleginnen und Kollegen habe ich dann begonnen mich zunächst mit Traumatisierungen bei Menschen mit Psychosen zu beschäftigen. Als ich später meinen Schwerpunkt in den Suchtbereich verlagerte und als wissenschaftlicher Mitarbeiter im Zentrum für Interdisziplinäre Suchtforschung (ZIS) in Hamburg anfing zeigte sich wie groß die Bedeutung des Themas auch bei Suchtkranken ist.

\section{Welche Rolle spielt Traumatisierung bei der Entstehung von Suchtstörungen?}

Es verhält sich wie mit allen Risikofaktoren hinsichtlich der Ätiologie einer psychischen Störung. Bei einem Teil der Menschen mit Suchtstörungen spielen Traumatisierungen überhaupt keine Rolle. Etwa ein Drittel bis die Hälfte aller Suchtkranken musste Erlebnisse sexueller oder körperlicher Gewalt in der Kindheit machen, ein weiterer Teil er- lebte emotionale Vernachlässigung in der Vorgeschichte. Nicht bei allen Betroffenen müssen die Folgen dieser Erlebnisse bei der Suchtentwicklung eine bedeutsame Rolle gespielt haben. Bei einem großen Teil der Betroffenen haben sie deren Biografien aber so entscheidend geprägt, dass der Einstieg in die Suchtkarriere und oft auch die aktuelle Problematik stark mit den Folgen der traumatischen Erfahrungen zusammenhängt. Bei einer Untergruppe von Betroffenen sind diese Zusammenhänge so eng, dass man die Suchtstörung eigentlich als Traumafolgestörung verstehen sollte, mit Konsequenzen für den Umgang mit Betroffenen und die notwendigen therapeutischen Ansätze.

\section{Wie hängen diese beiden Störungsbilder (innerlich) zusammen?}

Besonders augenfällig sind die Zusammenhänge im Falle sogenannter komorbider Störungen, wie der Posttraumatischen Belastungsstörung, die sich bei 10 bis 30 Prozent aller stationär behandelten Patientinnen und Patienten findet, wenn eine systematische Diagnostik erfolgt. Der Begriff der Komorbidität ist dabei nicht nur hilfreich. Insbesondere wenn er suggeriert, dass es sich um letztlich parallel entstandene Probleme handelt. Es geht vielmehr um verschiedene Aspekte einer meist sehr komplexen Gesamtproblematik. Dass es sich beim Substanzkonsum um einen Weg des Umganges mit Traumatisierungen handelt, ist dann meist offensichtlich. Entgegen häufigen Stereotypen geht es dabei nicht immer nur um Symptome der Posttraumatischen Belastungsstörung, die bei Suchtkranken zumeist mit Erlebnissen körperlicher und sexueller Gewalt in der Kindheit zusammen hängen. Oft dominieren Störungen der Emotionsregulation, Schwierigkeiten in Beziehungen und ein sehr negatives Selbstbild, alles Beschwerden die typischer Weise nach frühen Traumatisierungen auftreten, auch wenn es sich dabei um emotionale Gewalt oder Vernachlässigung gehandelt hat.
Welche Therapiemöglichkeiten sind beim gleichzeitigen Auftreten von Sucht und Traumastörungen wirksam und deshalb zu empfehlen?

Weil Gewalt und Vernachlässigung in den Biographien Suchtkranker so häufig sind, sollte für den Umgang mit ihnen und in der Behandlung generell die Trauma-Perspektive eine weit größere Rolle einnehmen als bisher. Wir wissen, dass Menschen, die in ihrer Kindheit von nahen Bezugspersonen sehr Belastendes erleben mussten, häufig dadurch nachhaltig geprägt sind. Dies spiegelt sich dann in ihren Interaktionen im $\mathrm{Er}$ wachsenenalter wieder.

Beim Umgang mit den Betroffenen sollten deshalb wichtige Aspekte berücksichtigt werden, die allgemein bei Menschen mit Traumatisierungen wichtig sind. Dazu gehört es unter anderem traumatische Erfahrungen anzusprechen und nicht abzuwarten ob Betroffene diese spontan berichten, ihre Sicht darauf zu validieren und Informationen zu Hilfsmöglichkeiten anzubieten. Weiter müssen in die Suchthilfe Interventionen integriert werden, deren Wirksamkeit in Bezug auf die Folgen traumatischer Erfahrungen inzwischen gezeigt ist. Bei Vorliegen von Traumafolgestörungen wie der PTBS gehören dazu auch traumafokussierte Therapien, die bislang nur ein Bruchteil der Betroffenen erhält. Obwohl es auch gelingen kann beide Problembereiche parallel durch jeweils spezialisierte Therapeutinnen zu behandeln sollte einer integrativen Behandlung der Vorzug gegeben werden. Dabei geht es aus meiner Sicht vor allem um die Integration von traumatherapeutischen Ansätzen in die Suchthilfe, deren Kompetenzen schwerer an anderer Stelle zu implementieren sind als das Wissen um den Umgang mit Traumatisierungen im Suchtbereich. Die Idee, dass erst bei stabiler Abstinenz traumatherapeutische Interventionen sinnvoll sind ist in jedem Fall obsolet. 
Sie haben das bundesweite Forschungsprojekt CANSAS initiiert und koordiniert. Was sind die wichtigsten Erkenntnisse daraus?

Es ging bei diesem multizentrischen Forschungsprojekt unter Förderung des Bundesministeriums für Bildung und Forschung (BMBF) darum, die Zusammenhänge zwischen früher Traumatisierung und Sucht und die Weitergabe beider Phänomene an die nächste Generation zu erhellen. Außerdem sollten therapeutische und präventive Strategien für traumatisierte Suchtkranke und ihre Kinder entwickelt und erprobt werden. Es ging also neben grundlegenden Erkenntnissen zu den Mechanismen, wie Trauma und Sucht über Generationen zusammenhängen, vor allem um neue Therapien und die traumaspezifische Weiterentwicklung des Hilfesystems. Es zeigte sich, dass stabilisierende Therapien gut akzeptiert werden, sie aber bei Suchtkranken mit PTBS nicht ausreichen und um expositionsbasierte Verfahren ergänzt werden sollten. Das bereits vorliegende Programm „Sicherheit finden " konnte erfolgreich evaluiert und erweitert werden.

Im Bereich Kinderschutz ergab sich ein relevanter Bedarf an strukturierten Werkzeugen zur Einschätzung von Risiken. Hier wurde mit dem „Hamburger Gefährdungsbogen“ ein Screening-Instrument zur Risikoabschätzung entwickelt und evaluiert. Weiter ging es darum, Fachkräften, die mit Suchtkranken arbeiten, mit dem Programm „Learning how to ask“ ein brauchbares Werkzeug zur Erhebung von Traumatisierungen und zum richtigen Umgang damit zur Verfügung zu stellen.

Gibt es Zusammenhänge zwischen bestimmten Traumatisierungsformen und einzelnen bevorzugten Substanzen und dementsprechend verschiedenen Suchtformen?

Lange Zeit war man davon ausgegangen, dass dies so ist, dass etwa die vegetative Überregung bei traumatisierten Menschen oder die sich aufdrängenden Erinnerungen zu einer Affinität gegenüber sedierenden Substanzen führt. Dies ist auch oft der Fall, aber es ist keineswegs ein zwingender Zusammenhang. Offenbar spielt die Verfügbarkeit von Substanzen eine dominante
Rolle und das generelle Bestreben nach Zustandsveränderung. So kann es nach starker Traumatisierung genauso zu einem chronischen Alkoholmissbrauch wie zu einem polyvalenten Konsum von Substanzen kommen. Auch Stimulantien können dabei beteiligt sein, etwa um eine Stimmungsaufhellung zu erreichen.

Welche Empfehlungen gibt es für die traumainteressierte Suchthilfepraxis?

Neben den schon erwähnten Innovationen zum Screening und der Behandlung der betroffenen Patientinnen und Patienten in traumasensiblen Programmen ist es vor allem die Offenheit für das Thema, welche Praktikern die Arbeit erleichtern, und nicht wie oft fälschlicherweise angenommen, erschweren kann. Durch traumaspezifisches Wissen und Handeln können Therapeutinnen und Therapeuten ihre Arbeit erleichtern und bereichern. Die Verhaltensweisen Betroffener machen unter einer traumasensiblen Perspektive zumeist Sinn, finden dadurch eine andere Bewertung und in der Folge andere therapeutische Reaktionen. Gerade bei Suchtkranken, die immer wieder Probleme mit der Emotionsregulation, ihrer Abstinenzmotivation, einer guten Selbstfürsorge, selbstverletzendem Verhalten und Rückfallvermeidung haben, stehen häufig Traumatisierungen im Hintergrund. Wenn sie in die Fallkonzeption einbezogen werden kann ein besseres Verständnis für die aktuellen Probleme auf beiden Seiten und neue Motivation entstehen. Die oft geäußerte Befürchtung vor einer schwerwiegenden Re-Traumatisierung ist zumeist unberechtigt. Die Risiken, die sich aus dem Unterlassen therapeutischer Unterstützung ergeben sind meist viel schwerwiegender. Kurz lässt sich zusammenfassen: Suchttherapeuten sollten keine Berührungsängste mit dem Thema Trauma haben, spezifisches Wissen dazu ist leicht zu erwerben und es stehen effektive Hilfsmöglichkeiten zur Verfügung.

Wie sollte eine gemeinsame, koordinierte Therapie für Sucht- und Traumastörungen aussehen?

Inzwischen liegt eine Vielzahl von Therapiemanualen und -ansätzen für traumatisierte Suchtkranke vor. Die verschiedenen Programme, sei es „Sicherheit finden“, „DBT-
Sucht" oder traumafokussierte Verfahren, wie die prolongierte Exposition oder die Narrative Expositions-Therapie, sollten Standardangebote in jeder Suchttherapieeinrichtung sein. Wichtig ist nunmehr, dass dieses Wissen und die vorhandene Kompetenz in die Fläche kommen. Die Vielzahl der Suchtkranken, bei denen Traumatisierungen und ihre Folgen einen bedeutsamen Einfluss auf die Suchterkrankung haben, wird davon profitieren.

Ist eine Prävention von Suchterkrankungen bei Menschen mit Traumaerfahrungen möglich?

Im Prinzip ist diese Perspektive entscheidend. Gerade im Bereich traumatisierter Kinder, und hier ist nicht nur an sexuelle und physische Misshandlung, sondern auch an emotionale Vernachlässigung zu denken, stellen sich im Vorfeld des Substanzkonsums bereits relevante Störungen ein, etwa der Emotionsregulation. Diesen Kindern und Jugendlichen könnte frühzeitig mit übenden Verfahren, wie z. B. „Gefühle im Griff“ von Prof. Sven Barnow geholfen werden.

\section{Wir danken Ihnen für das Gespräch.}

Autorinnen/Autoren

Prof. Dr. med. Ingo Schäfer, MPH, Psychiater und Psychotherapeut, Leiter Arbeitsbereich Suchtmedizin und Abhängiges Verhalten, Universitätsklinikum Hamburg-Eppendorf, Direktor, Zentrum für Interdisziplinäre Suchtforschung (ZIS) der Universität Hamburg, Past-Präsident, Deutschsprachige Gesellschaft für Psychotraumatologie (DeGPT), Präsident, European Society for Traumatic Stress Studies (ESTSS). 